\title{
Helicobacter pylori: una causa no tradicional de deficiencia de hierro y anemia
}

\author{
CAROLINA SERRANO H. ${ }^{1}$, ANDREA VILLAGRÁN T. ${ }^{1}$, PAUL R. HARRIS D. ${ }^{1}$ \\ 1. Unidad de Gastroenterología y Nutrición Pediátrica, División de Pediatría, Facultad de Medicina. \\ Pontificia Universidad Católica de Chile.
}

\begin{abstract}
Helicobacter pylori: a non traditional cause of iron deficiency and anemia

Helicobacter Pylori (H. pylori) is the most common cause for chronic gastritis, duodenal ulcers, MALT lymphoma and gastric cancer. H. pylori has also been associated with several extra gastric diseases such as iron deficiency (ID) and iron deficiency anemia (IDA). Several mechanisms by which $H$. pylori might contribute to the development of ID/IDA have been proposed. Alterations in the physiology of gastric mucosa and of iron metabolism as a consequence of the infection as well as direct competition for iron by $H$. pylori have been proposed as possible causes for ID/IDA development. H. pylori causes alterations in gastric $\mathrm{pH}$ as well as in the concentration of ascorbic acid in the stomach limiting the absorption of dietary iron in the gastrointestinal tract. In addition, pro-inflammatory cytokine secretion in response to the infection, in particular IL-1 $\beta$ are able to modulate acid secretion and stimulate the secretion of hepcidin from the liver inhibiting liberation of iron from iron stores. These results in less iron available for eritropoyesis, thus contributing to ID/IDA development. Several studies have suggested a direct relationship between the presence of the infection and the development of ID/IDA in children, adolescents and adults. H. pylori eradication has been associated to a better response to oral iron therapy in IDA patients. However some controversy still remains regarding the role of the infection in ID/IDA and the mechanisms involved. Studies aimed to evaluate the relationship between H. pylori and acid output in gastric mucosa, as well as studies to determine iron capture in strains isolated from IDA patients are still being conducted in order the shed some definitive light in the relationship between this bacterium and ID/IDA and their contributing mechanisms.
\end{abstract}

(Key words: Helicobacter pylori, iron deficiency, iron uptake).

Rev Chil Pediatr 2012; 83 (1): 13-23

\section{RESUMEN}

Helicobacter pylori, es la causa más común de gastritis crónica y está etiológicamente asociado con úlcera duodenal y gástrica, adenocarcinoma gástrico y linfoma tipo MALT. Adicionalmente, ha sido asociado con varias patologías extra gástricas siendo la anemia por deficiencia de hierro (IDA) y su etapa previa, la ferropenia o deficiencia de hierro (ID), una de las más recientes y poco exploradas. El mecanismo biológico mediante el cual la infección por $H$. pylori contribuye al desarrollo de ID/IDA es multifactorial y comprende en parte

Trabajo recibido el 13 de diciembre 2011, aceptado para publicación el 24 de enero de 2012.

Financiamiento: Fondecyt 1100654, Proyecto CONTENT 6th FP de la Unión Europea (INCO-DEV-3-032136), y Conicyt/BM RUE\#29.

Correspondencia a:

Dr. Paul R. Harris D.

E-mail: pharris@med.puc.cl 
alteraciones provocadas en la fisiología de la mucosa gástrica como consecuencia de la infección, alteraciones en el metabolismo del hierro, como también competencia directa por el hierro biodisponible en el estómago por parte de la bacteria. Alteraciones provocados en el pH gástrico y en la concentración de ácido ascórbico por la presencia de $H$. pylori pueden limitar la absorción del hierro dietario en el tracto gastrointestinal. Adicionalmente se ha descrito que la secreción de citoquinas proinflamatorias, particularmente IL-1 $\beta$ es capaz de modular la secreción de ácido y estimular la liberación del péptido hepcidina desde el hígado. Este último inhibe la liberación de hierro desde sus reservorios celulares como macrófagos y eritrocitos resultando en una disminución del hierro disponible para la eritropoyesis contribuyendo al desarrollo de ID/IDA. Varios estudios han sugerido una asociación directa entre anemia por déficit de hierro y la infección por $H$. pylori en niños, adolescentes y adultos. La erradicación del $H$. pylori se asocia a una mejor respuesta a la terapia oral de hierro en comparación con el uso de la terapia de hierro por sí sola. Sin embargo, hay controversia sobre la eficacia de la erradicación de la infección por $H$. pylori en el tratamiento de ID/IDA, así como de los mecanismos involucrados. Estudios dirigidos a evaluar la relación entre ID/IDA en sujetos infectados por $H$. pylori y la secreción de ácido en el estómago y captación de hierro por las cepas infectantes, se encuentran actualmente en curso con el propósito de elucidar de manera clara el rol y los mecanismos mediantes los cuales esta bacteria contribuye al desarrollo de ID/IDA. En este artículo presentamos los últimos avances en el conocimiento sobre el rol de Helicobacter pylori en la génesis de déficit de hierro.

(Palabras clave: Helicobacter pylori, deficiencia de hierro, captación de hierro).

Rev Chil Pediatr 2012; 83 (1): 13-23

\section{Historia natural de la infección}

Helicobacter pylori (H. pylori) coloniza el estómago de aproximadamente la mitad de la población mundial. Esta infección adquirida en la infancia, tiene la capacidad de persistir durante décadas ${ }^{1}$. La infección es responsable de una parte no menor de la morbilidad y mortalidad asociada a infecciones del tracto digestivo. Patologías como la úlcera péptica, el linfoma MALT y el adenocarcinoma gástrico han sido relacionadas con la presencia de H. pylori y elevado a este último al estatus de carcinógeno de tipo $\mathrm{I}^{2}$.

La prevalencia de la infección es mayor en los países en desarrollo. En Chile, el 73\% de la población adulta presenta títulos de anticuerpos contra $H$. pylori compatibles con la presencia de la bacteria ${ }^{3}$. Adicionalmente esta infección es considerada como un factor de riesgo de mortalidad y de pérdida de años de vida saludable por el Ministerio de Salud ${ }^{4}$. El cáncer gástrico es también la causa más importante de muerte por cáncer en hombres en nuestro país con una tasa de mortalidad 19/100 000 habitantes $^{3-5}$.

La adquisición de la infección por $H$. pylori está relacionada con el nivel socioeconómico y las condiciones de vida del hospedero, a través de una vía directa (oral). Específicamente el hacinamiento, la calidad del agua para el consumo doméstico, la falta de servicios higiénicos, la pertenencia a regiones con mayores niveles de ruralidad, ingresos familiares bajos y bajos niveles educacionales se han correlacionado históricamente con un aumento en la infección por H. pylori ${ }^{6}$.

La generación y magnitud del daño asociado a esta infección depende de la ocurrencia de eventos inmunes que persiguen la erradicación de la bacteria, junto con la presencia de factores de virulencia bacterianos. Habitualmente la mayoría de la población infectada con $H$. pylori sólo desarrolla una gastritis crónica leve asintomática. De los infectados un $10-15 \%$ desarrolla úlceras gastroduodenales. Porcentajes aún menores del total de los infectados desarrollan linfoma de tipo MALT y adenocarcinoma del estómago. En general los cursos clínicos son excluyentes y existen datos consistentes que avalan que el desarrollo de úlcera duodenal, no así la úlcera gástrica, es un factor protector para el desarrollo del cáncer (figura 1$)^{7}$. 


\section{H. pylori y enfermedades extragástricas}

Además de su bien demostrado rol en el desarrollo de enfermedades gastroduodenales, y similar a lo que se ha propuesto para otros agentes infecciosos crónicos, se ha postulado una relación entre $H$. pylori y una serie de enfermedades extragástricas como por ejemplo, cardiopatía isquémica, púrpura trombocitopénico idiopático, anemia por deficiencia de hierro (IDA), y otros trastornos. La evidencia para estas asociaciones es de diversa naturaleza, la mayoría basada en descripciones de casos clínicos y en algunos casos de estudios epidemiológicos y dan cuenta de cómo una infección crónica localizada es capaz de incidir en el desarrollo de condiciones patológicas concurrentes y de carácter sistémico ${ }^{8}$. Una larga lista de otras asociaciones que se han acumulado en el tiempo, no han logrado ser documentadas consistentemente en cuanto al papel de $H$. pylori en su etiología.

\section{H. pylori y deficiencia de hierro}

\section{Deficiencia de hierro y anemia ferropriva}

La deficiencia de hierro (ID) resulta como consecuencia de un balance de hierro negativo donde los depósitos de almacenaje de hierro disminuyen progresivamente y la cantidad de hierro disponible para su transporte en el plasma y posterior utilización en los tejidos se encuentra comprometida. En su etapa más avanzada la deficiencia de hierro lleva al desarrollo de anemia (IDA). La ID/IDA es el desorden nutricional más común en el mundo. Se estima que alrededor de un billón de niños con un rango de edad de 0-14 años padece de anemia ferropriva. En países en desarrollo la prevalencia de la anemia varia desde un 39\% (edad 0-4) a un $48 \%$ (edad 5-14) $)^{9}$. Las manifestaciones clínicas de ID/IDA incluyen alteraciones en el desarrollo físico y cognitivo. Se ha demostrado en niños un retraso del desarrollo psicomotor, medido como un deterioro del rendimiento en

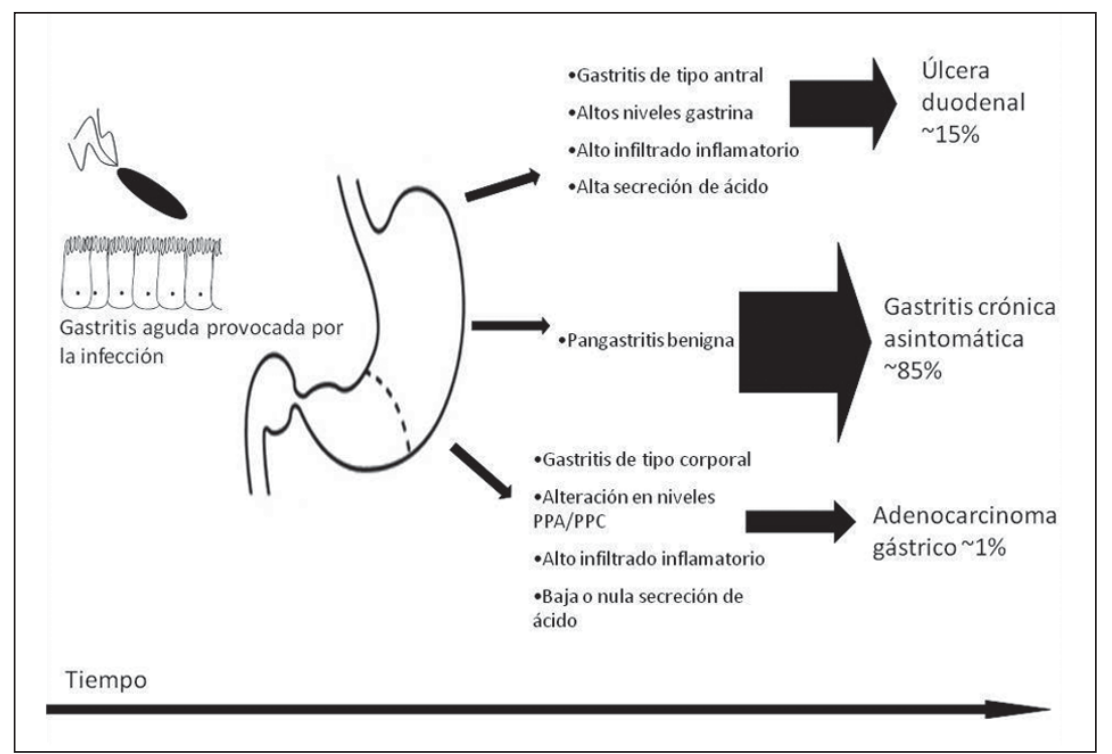

Figura 1. Historia natural de la infección por $\boldsymbol{H}$. pylori. La infección por $H$. pylori genera en primera instancia una gastritis aguda asintomática que progresa con el tiempo a uno de los tres grandes tipos de gastritis. El fenotipo más común se caracteriza por una pangastritis moderada, sin alteraciones en la secreción de ácido y la presentan personas asintomáticas que no desarrollan complicaciones serias. El segundo fenotipo se asocia con la aparición de úlceras duodenales y se caracteriza por una gastritis de predominio antral con niveles elevados de gastrina e infiltrado inflamatorio y alta secreción de ácido que se presenta aproximadamente en un $15 \%$ de los infectados. El tercer fenotipo se caracteriza por una gastritis de predominio corporal, alteraciones en la razón de pepsinogenos, atrofia gástrica multifocal y bajos niveles de secreción de $\mathrm{HCl}$ y se considera como una un factor de riesgo en el desarrollo de cáncer gástrico. Se presenta en un porcentaje cercano al 1\% de los infectados. La presencia de uno de los fenotipos suele ser excluyente de los otros. 
las pruebas de lenguaje, de habilidades, destrezas motoras y de coordinación en varios países de Latinoamérica y el mundo ${ }^{9-12}$.

Otros efectos deletéreos asociado a la ID/ IDA en adultos son un aumento de la morbilidad y mortalidad por enfermedades infecciosas asociada una disminución en la función inmune $^{13,14}$, alteraciones en la producción de triyodotironina y la función tiroidea en general, además de la producción y el metabolismo de las catecolaminas y de otros neurotransmisores involucrados en la mantención del metabolismo y la regulación de la temperatura corpora $^{15,16}$. Los antecedentes anteriores implican que una correcta captación y transporte de hierro es de vital importancia para el desarrollo normal de la población y que las poblaciones en desarrollo son especialmente susceptibles a los efectos patológicos de la ID/IDA.

\section{Evidencia clínica de asociación entre ID/ IDA y la infección por $\mathrm{H}$. pylori}

En 1993, Dufour publicó el primer reporte, en un niño, describiendo la relación entre ID o IDA refractaria a tratamiento convencional con suplementación de hierro, que fue revertida sólo posterior a la erradicación de H. pylo$r i^{17}$. Posterior a ello, se observó un aumento de reportes clínicos aislados y pequeñas series clínicas en adolescentes y adultos corroborando esta asociación ${ }^{18-20}$. Dichos estudios reportaron disminuciones en los niveles de ferritina sérica en pacientes seropositivos para la infección por H. pylori, aumentos en la prevalencia de la infección en grupos con anemia por deficiencia de hierro, y a $H$. pylori como factor de riesgo para un crecimiento disminuido en adolescentes ${ }^{21-24}$. La mayor parte de estos estudios fueron descriptivos, estudios epidemiológicos con determinación no invasiva (serológica) de la infección por H. pylori, y han sido revisadas extensamente ${ }^{25}$.

Adicionalmente, reportes de series de casos en otros grupos etáreos describiendo la mejoría de ID/IDA luego de la erradicación de la bacteria aparecieron en la literatura y el primer estudio clínico doble ciego controlado (RCT) con erradicación terapéutica de $H$. pylori en pacientes adolescentes con ID fue publicado por Choe y cols. De los pacientes incluidos en ese estudio un grupo recibió suplementación oral de hierro, otro grupo tratamiento de antibióticos y un tercer grupo tanto hierro oral como antibióticos para erradicar la bacteria. De los pacientes analizados, sólo los grupos que recibieron tratamiento con antibióticos demostraron una mejoría en los niveles de hemoglobina independientemente de si habían recibido suplementación oral con hierro ${ }^{26}$.

A partir de la década del 2000, aparecieron los primeros estudios negativos, que no lograron reproducir estos primeros hallazgos ${ }^{27-29}$. Sin embargo, simultáneamente la literatura persistió reportando series clínicas y estudios transversales serológicos, demostrando la relación entre ID/IDA y $H$. pylori en diferentes poblaciones etárea y áreas geográficas ${ }^{30-33}$.

Gran parte de esta aparente discordancia en los estudios mencionados anteriormente derivan de a los menos 5 variables importantes, a mencionar: i) la mayoría de los estudios no evaluó la presencia de co-morbilidad ya sea porque no descartaron patología infecciosa asociada o por ausencia de estudios endoscópicos/histológicos del duodeno; ii) la mayoría de los estudios no evaluó el uso simultáneo de medicamentos del tipo inhibidores de bomba de protones, anti $\mathrm{H}_{2}$, u otros que alteran profundamente la secreción gástrica y por ende la fisiología de la absorción de hierro; iii) hubo una gran heterogeneidad en las poblaciones evaluadas, tanto por edad como por área geográfica; iv) la mayoría de los estudios descriptivos, epidemiológicos e incluso los RCT no contaron con evaluación endoscópica de la infección por H. pylori o de la indemnidad duodenal, y el diagnóstico de $H$. pylori fue sobre bases serológicas, test de urea en aire espirado $\mathrm{u}$ otros igualmente no invasivas, $\mathrm{y} v$ ) finalmente, la mayoría de los estudios no estudió el $\mathrm{pH}$ gástrico u otras variables fisiológicas que alteran la absorción de hierro y por tanto, son determinantes en el nivel y mantención de la ID.

La aparición de los primeros estudio randomizados de erradicación el 2006 hizo pensar en una rápida resolución en la discordancia clínica antes referida. Dos estudios iniciales contaron con un alto número de niños y adolescentes (mayor a 600 sujetos cada uno) demostraron resultados parcialmente opuestos ${ }^{29,34}$. 
A la fecha, más de 15 RCT han sido publicados. Ellos son mejor analizados en 3 recientes metanálisis. En el primer metanálisis publicado en 2010, de 237 potenciales artículos, y luego de las exclusiones, sólo 16 cumplieron satisfactoriamente los criterios de inclusión. En breve, los pacientes que recibieron terapia antibiótica anti- $H$. pylori más hierro $(\mathrm{n}=494)$ comparados con aquellos que recibieron sólo hierro $(\mathrm{n}=462)$, presentaron una más rápida recuperación en el nivel de hemoglobina, independiente del nivel inicial de hemoglobina (diferencias entre medias: 1,48 g/dl, IC 95\%: 0,96-2,00, $\mathrm{p}<0,00001)$. Del mismo modo, el efecto sobre la recuperación en el nivel de ferritina fue significativamente superior en el grupo que recibió antibióticos más hierro comparado con aquellos que sólo recibieron hierro (diferencias entre medias: 1,84 $\mu \mathrm{g} / \mathrm{L}$, IC 95\%: $1,2-2,48, \mathrm{p}<0,00001)$. Es necesario destacar que la mayor parte de los estudios provinieron de Oriente con 12 manuscritos de China, y uno de Corea del Sur, India, EE.UU., y Bangladesh $^{35}$.

El segundo metanálisis, publicado también el 2010, reportó resultados de 12 RCT analizados a partir de una búsqueda de más de 45 potenciales RCT. Sólo 3 nuevos artículos no estaban presentes en el primer metanálisis. El estudio concluyó que la erradicación de $H$. pylori aceleró la mejoría en los niveles de ferritina en sujetos con ID (diferencia entre medias: 7,74 $\mu \mathrm{g} / \mathrm{L} ; 95 \% \mathrm{CI}: 4,61-10,88 ; \mathrm{p}<0,00001$ ), y también aceleró la mejoría en los niveles de hemoglobina a los 2 meses de tratamiento (diferencia entre medias: $1,96 \mathrm{~g} / \mathrm{dl}$; $95 \% \mathrm{CI}$ : 1,48$2,44 ; \mathrm{p}<0,00001)$ y a un año después de iniciado el tratamiento (diferencia entre medias: $0,37 \mathrm{~g} / \mathrm{dl} ; 95 \%$ CI: 0,08-0,65; $\mathrm{p}=0,01)^{36}$.

Un tercer metanálisis relevante, a partir de 1267 potenciales artículos analizó 8 RCT, de los cuales 6 se incluyeron en el primer metanálisis y 6 en el segundo. Por ello, no es sorprendente que sus autores demuestren que el nivel de hemoglobina se corrige favorablemente después de la erradicación bacteriana más hierro comparada con un grupo que sólo recibió hierro, tanto en niños, pero mayoritariamente en adultos (diferencias entre medias: 12,43 g/ dl, IC 95\%: 5,23-19,62, p < 0,0007). El nivel de ferritina siguió la misma tendencia con mayor recuperación en adultos que en niños (diferencia entre medias: $9,55 \mu \mathrm{g} / \mathrm{L}$; $95 \% \mathrm{CI}$ : 5,07$14,03 ; \mathrm{p}<0,0001)^{37}$.

En resumen, la mayoría de los estudios muestran una relación entre la infección por H. pylori e ID/IDA. El grado de correlación parece estar asociado con la edad avanzada y la duración de la anemia. Algunos estudios de intervención muestran una mejora en los parámetros hematológicos de hierro sólo después de erradicación de H. pylori. La falta de asociación entre $H$. pylori e ID en algunos estudios puede explicarse por patología gastrointestinal simultánea o medicamentos, las diferencias en la evaluación de $H$. pylori y las diferencias regionales en las poblaciones estudiadas. Es posible que las diferencias reportadas en la literatura sean resueltas en la medida que se desarrollen estudios clínicos que consideren dichas variables como co-morbilidad, normalidad histológica duodenal, homogenización en el diagnóstico de la infección (idealmente endoscópico) y evaluación de $\mathrm{pH}$ gástrico y otros parámetros fisiológicos que regulan la absorción de hierro.

\section{Mecanismos que generan ID}

Los mecanismos mediante los cuales $H$. pylori puede contribuir al desarrollo de deficiencia de hierro y posteriormente a la anemia son múltiples y de diversa naturaleza. Existen mecanismos generalizados que son parte de la respuesta inflamatoria del organismo, y también mecanismos más específicos asociados a la infección por $H$. pylori propiamente tal. Durante la inflamación, la hepcidina, liberada por el hígado como proteína de fase aguda en respuesta a dicha inflamación, inhibe la liberación de hierro de los macrófagos y los enterocitos. Dado que la mayoría de hierro unido a la transferrina es destinado a la médula ósea, existe una considerable disminución de hierro disponible para la eritropoyesis lo que puede traducirse en la instauración de anemia en respuesta a la hipoferremia generada por la inflamación. Además, la hepcidina puede contribuir a la anemia de la inflamación no sólo a través de efectos sobre el metabolismo del hierro, sino también a través de la inhibición de la 
proliferación celular y la supervivencia de los progenitores eritroides ${ }^{38,39}$. En el caso especifico de la infección por $H$. pylori, no ha sido posible detectar un aumento sistemático en los niveles séricos tanto de pro-hepcidina como de hepcidina en los pacientes infectados con $H$. pylori. Alteraciones en la hepcidina correlacionan con la presencia de IDA de manera independiente de la infección ${ }^{40,41}$. Recientemente se ha reportado la expresión de hepcidina en la mucosa gástrica tanto de murinos como humanos. La presencia de $H$. pylori es además capaz de inducir la expresión de ésta en células AGS, una línea celular derivada de un adenocarcinoma gástrico. Si bien los niveles séricos tanto de hepcidina como pro-hepcidina parecen no estar alterados en los pacientes infectados con respecto a aquellos que no lo están, la expresión de dicha molécula en tejido gástrico si se ve alterada en presencia de la infección disminuyendo luego de una erradicación exitosa. Esta expresión local de hepcidina al parecer estaría regulada de manera independiente de la hepcidina hepática y entre sus efectos estaría la regulación de la secreción de ácido ${ }^{42}$.

La infección por H. pylori genera además infiltración por parte de polimorfonucleares neutrófilos al sitio de la infección. Estos secretan moléculas que acomplejan hierro como la lactoferrina, generando un estado de baja disponibilidad de hierro en el organismo que tiene como objeto limitar el hierro disponible para el patógeno evitando que este sea capaz de colonizar y mantener una infección exitosa del hospedero ${ }^{43}$. En pacientes con ID infectados con $H$. pylori los niveles de lactoferrina en la mucosa gástrico se encuentran elevados. La erradicación de la bacteria normaliza los niveles de dicha proteína a medida que aumentan los niveles de hemoglobina ${ }^{44}$.

Por otro lado, H. pylori, contribuye al desarrollo de ID mediante la generación de disturbios en la fisiología de la mucosa gástrica como consecuencia de la infección. El pH ácido intragástrico juega un papel fundamental en la absorción de hierro a nivel del tracto digestivo. La principal fuente hierro del organismo proviene de la dieta. Del hierro dietario ingerido (1-2 mg/día) por un ser humano, el $20 \%$ se encuentra disponible acomplejado a grupos heme y es de origen animal, es soluble en el tracto gastrointestinal y es absorbido esencialmente en el duodeno. El 80\% del hierro restante, es de origen vegetal y se encuentra en forma no soluble como hierro férrico (Fe (III)). El transporte a través del intestino requiere de la reducción del metal a su estado ferroso (Fe (II)), y es en este proceso en que el ácido clorhídrico $(\mathrm{HCl})$ del estómago juega un rol de vital importancia. El ácido ascórbico, que se encuentra concentrado en el jugo gástrico, también contribuye formando un complejo con el Fe (III) evitando que este precipite y se pierda en el tracto gastrointestinal. Adicionalmente juega un rol en la solubilización y reducción del Fe (III) a Fe (II), proceso que depende del $\mathrm{pH}$ del estómago, por lo que alteraciones en la secreción de ácido gástrico pueden incidir en una captación reducida de este metal en el tracto gastrointestinal (figura 2) ) $^{45-47}$.

$H$. pylori genera un aumento en la expresión de citoquinas proinflamatorias como IL$1 \beta$, y TNF- $\alpha$ en la mucosa del estómago ${ }^{48}$. Ambas citoquinas son consideradas potentes inhibidoras de la secreción de ácido gástrico. Estudios in vitro demuestran que tanto IL- $1 \beta$ como TNF- $\alpha$, son capaces de inhibir la secreción de ácido e inducir apoptosis en células parietales como también disminuir la secreción de histamina por parte de las células tipo enterocromafines ${ }^{49-52}$. Adicionalmente se ha descrito la presencia de polimorfismos en el cluster génico de IL-1. Los sitios polimórficos incluyen tres sitios en la región promotora del gen de IL-1 $\beta$ en las posiciones +3 954, -511 (transición C-T), y -31 (transición T-C) y repeticiones variables en tándem de 86 pares de bases que dan origen a 5 alelos conocidos para el gen del antagonista del receptor de IL-1. La presencia de determinados alelos se asocian con un aumento en la expresión de esta citoquina en respuesta a infección por $H$. pylori, y es mediante este mecanismo dependiente de la expresión de citoquinas que llevan al desarrollo de hipoclorhidria, que $H$. pylori puede contribuir al deterioro de la absorción de hierro en el tracto gastrointestinal. Si bien la presencia de polimorfismos en el cluster de IL- $1 \beta$ ha sido asociada con la presencia de hipoclorhidria e históricamente han sido considerados como 


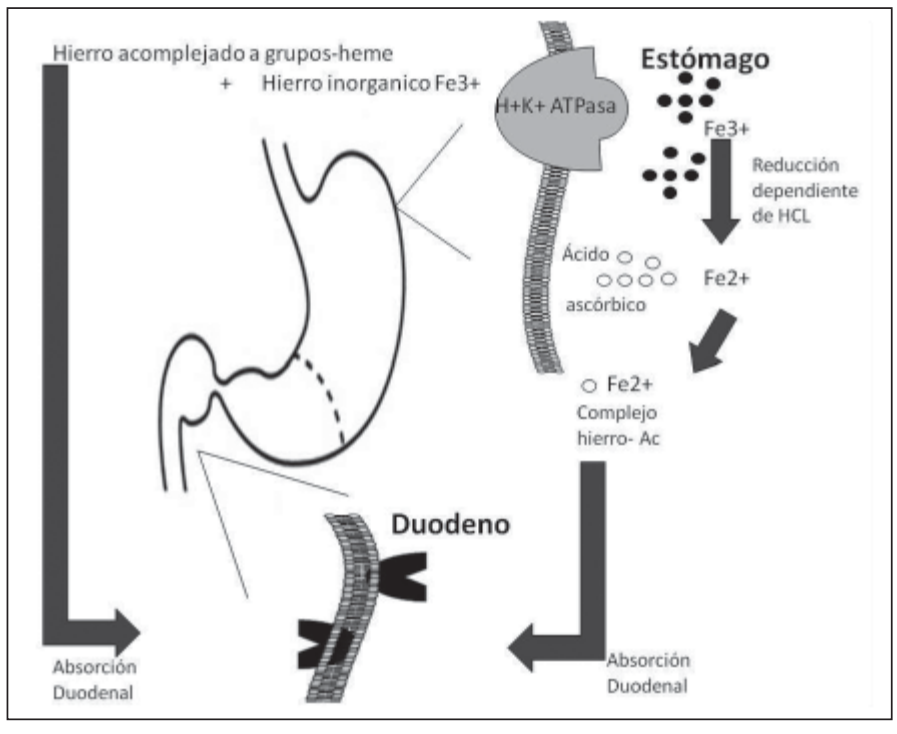

Figura 2. Absorción de hierro en el tracto digestivo. El $20 \%$ del hierro dietario se encuentra acomplejado a grupos heme, es soluble en el tracto gastrointestinal y se absorbe rápidamente en el duodeno. El 80\% restante se encuentra en forma no soluble como hierro férrico (Fe (III)). El transporte a través del intestino requiere de la reducción del metal a su estado ferroso (Fe (II)), en presencia del ácido clorhídrico $(\mathrm{HCl})$ Adicionalmente, el ácido ascórbico, también presente en el jugo gástrico, contribuye formando complejos con el Fe (III) evitando que este precipite y se pierda en el tracto gastrointestinal. un factor de riesgo en el desarrollo del cáncer gástrico no existe mucha información acerca del posible rol que estos polimorfismos pueden jugar en cuanto a su capacidad de funcionar como factores de riesgo para el desarrollo de ID en pacientes colonizados por $H$. pylori ${ }^{53}$.

Adicionalmente a los polimorfismos en IL$1 \beta$, existen polimorfismos en la región promotora del gen para TNF- $\alpha$, que han sido asociados también con un aumento de la expresión de dicha citoquinas. Atkinson y cols han asociado el cambio de base de $\mathrm{G}$ a A en la posición -308 del promotor de TNF- $\alpha$ con el desarrollo de anemia por deficiencia de hierro en presencia de otras enfermedades infecciosas como gatillantes como la malaria ${ }^{54}$.

Por otra parte, $H$. pylori tiene requerimientos altos de hierro para asegurar su colonización y permanencia en el estómago por lo que expresa un complejo sistema de captación y almacenamiento de hierro altamente regula$\mathrm{do}^{55,56}$. Dado que sólo una proporción de los infectados desarrolla ID y posteriormente anemia es posible que sean variaciones entre las cepas colonizantes con respecto a su capacidad de adquirir y almacenar hierro las responsables del desarrollo de la ID en un porcentaje de los pacientes infectados. H. pylori es capaz de utilizar las múltiples formas de presentación del hierro en el tracto digestivo. Posee sideróforos para la forma férrica del hierro presente en los alimentos y un transportador de membrana interna para la forma ferrosa. Adicionalmente $H$. pylori es capaz de crecer en la presencia de hemoglobina y otras proteínas con grupos heme como transferrina como la única fuente de hierro $^{57}$. Se ha reportado además la expresión de un receptor para lactoferrina ${ }^{44}$, subvirtiendo así, uno de los mecanismos clásicos antibacterianos utilizados por el hospedero para limitar la cantidad de hierro disponible a los patóge$\operatorname{nos}^{57,58}$.

Hasta la fecha, varios grupos de investigadores han demostrado diferencias entre las cepas de $H$. pylori aisladas de pacientes con ID/IDA en cuanto a su capacidad de captar hierro y el perfil general de expresión de proteínas dependientes de hierro, lo que sugiere que diferentes niveles de expresión de determinadas proteínas bacterianas en cepas particulares como las aisladas de los pacientes con IDA pueden estar contribuyendo directamente al desarrollo de la deficiencia de hierro en un grupo de pacientes en particular ${ }^{59,60}$. Un resumen de la función de algunos genes necesarios en la captación de hierro por la bacteria se presenta en la tabla 1.

La figura 3 resume los mecanismos potenciales dependientes de la bacteria (a la derecha de la figura) o del hospedero (a la izquierda de 
la figura) que podrían explicar la asociación y sus mecanismos plausibles entre $H$. pylori y el desarrollo de ID/IDA.

\section{Proyecciones y relevancia}

Los efectos deletéreos asociados a la deficiencia de hierro sobre todo en la población pediátrica tienen un impacto negativo de gran envergadura que frena el desarrollo de un país. Nuestro país ha implementado durante décadas programas de suplementación de nutrientes con el propósito de disminuir los efectos en el desarrollo de los niños asociadas a una pobre alimentación con resultados bastante significativos $^{61}$. Por lo demás, existen deficiencias nutricionales que encuentran entre sus agentes causantes algo más que sólo la mala ingesta asociada a situaciones socioeco- nómicas poco favorecedoras, siendo la IDA un ejemplo representativo de la asociación de una deficiencia nutricional con la presencia de una infección en particular, $H$. pylori. Nuestro país se encuentra además extensamente colonizado por esta bacteria con una prevalencia de la infección en población adulta de un $73 \%^{3}$, por lo que la influencia de esta infección sobre

Tabla 1. Genes asociados a la captación y almacenamiento de hierro en $\boldsymbol{H}$. pylori

\begin{tabular}{|ll|}
\hline Gen en estudio & Función conocida \\
\hline fecA1 & Sideróforo de hierro (Fe (III)) \\
fecA2 & Sideróforo de hierro (Fe (III)) \\
feoB & Transportador Fe (II) \\
frpB1 & Sideróforo de hierro (Fe (III)) \\
frpB2 & Receptor Hemoglobina \\
hugZ & Receptor Hemoglobina \\
\hline
\end{tabular}

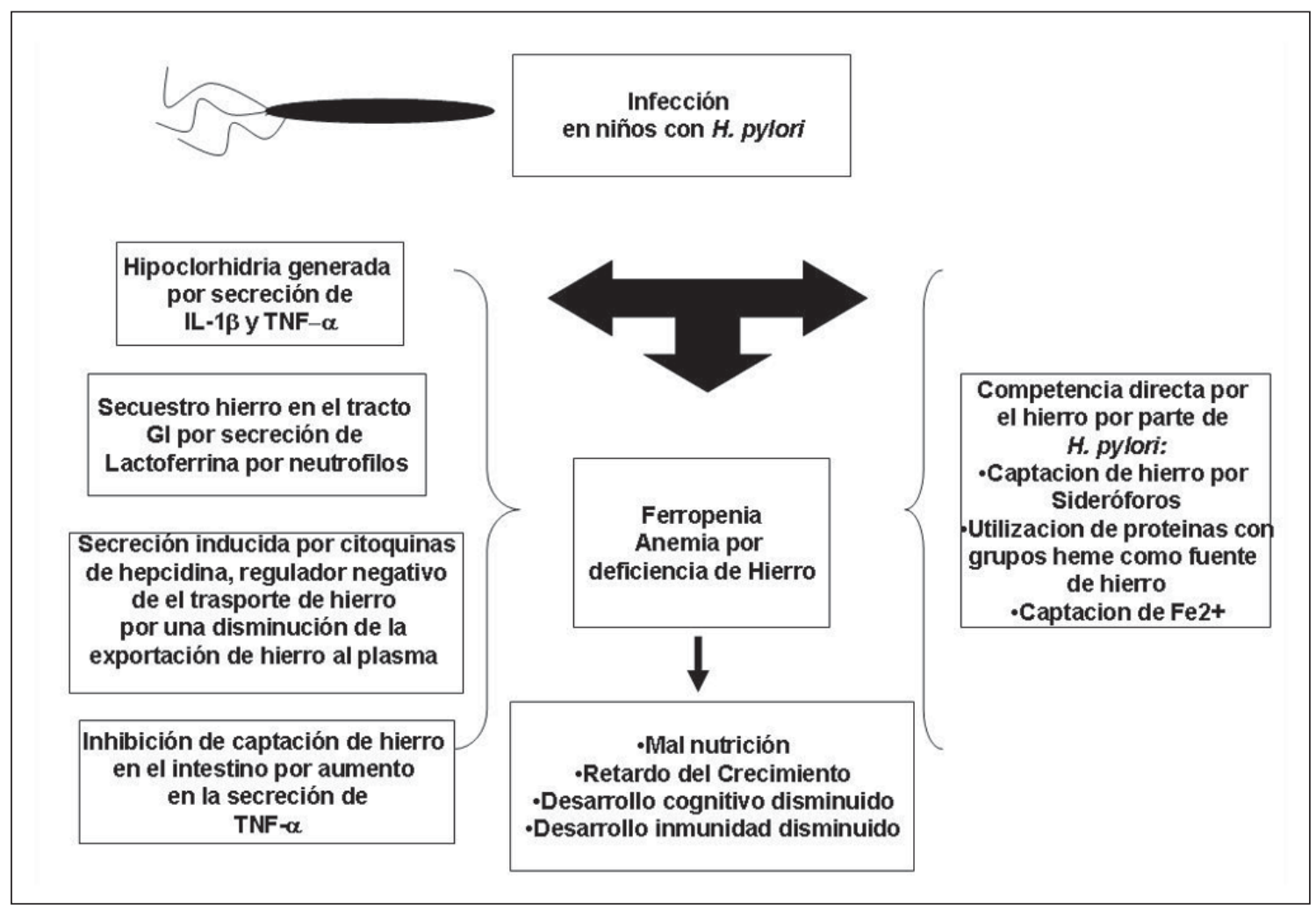

Figura 3. Efecto de la infección por en el metabolismo del hierro. H. pylori contribuye al desarrollo de ID/IDA al generar alteraciones en la fisiología de la mucosa gástrica, alteraciones en el metabolismo del hierro y competencia directa por el hierro biodisponible en el estómago por parte de la bacteria. 
cualquier condición que altera la salud de sus portadores es de vital importancia. Es relevante el poder establecer una vinculación entre la presencia de determinados alelos polimórficos de probada relación con el establecimiento de hipoclorhidria gástrica y una disminución en la captación de hierro en el estómago. Esta asociación ha sido tradicionalmente inferida pero rara vez comprobada. Adicionalmente $H$. pylori contribuye de manera directa compitiendo por los depósitos disponibles de hierro. Esta bacteria presenta un gran nivel de variabilidad inherente por lo que un estudio comprensivo de la dinámica de captación y almacenamiento de hierro acompañado de un estudio de los patrones de expresión de los genes asociados a estos procesos es vital en la mejor comprensión de cómo cepas con ciertas particularidades son capaces de generar condiciones patológicas sistémicas en respuesta a la infección por H. pylori.

\section{Referencias}

1.- Torres J, Pérez-Pérez G, Goodman KJ, et al: A comprehensive review of the natural history of Helicobacter pylori infection in children. Arch Med Res 2000; 31: 431-69.

2.- Cover TL, Blaser MJ: Helicobacter pylori in health and disease. Gastroenterology 2009; 136: 1863-73.

3.- Ferreccio C, Rollán A, Harris P, et al: Gastric Cancer is Related to Early Helicobacter pylori Infection in a High Prevalence Country. Cancer Epidem Biomar 2007; 16: 662-7.

4.- República de Chile. Ministerio de Salud: Departamento de Estadisticas Minsal. Estudio de Carga de enfermedad y Carga atribuible. 2007. Disponible en http://deis. minsal.cl/.

5.- República de Chile. Ministerio de Salud: Departamento de Estadísticas. Bases de datos de Mortalidad 19902004. Disponible en http://deis.minsal.cl/.

6.- Lehours P, Yilmaz O: Epidemiology of Helicobacter pylori infection. Helicobacter 2007; 12: Suppl 1: 1-3.

7.- Amieva MR, El-Omar EM: Host-bacterial interactions in Helicobacter pylori infection. Gastroenterology 2008; 134: 306-23.

8. Nilsson HO, Pietroiusti A, Gabrielli M, et al: Helicobacter pylori and extragastric diseases. Helicobacter 2005; 10: Suppl 1: 54-65.

9. Stoltzfus RJ: Iron deficiency anaemia: re-examining the nature and magnitude of the public health problem. J Nutr 131 2001; 697S-700S.

10. Walter T, Kovalsys J, Stekel A: Effect of mild iron deficiency on infant mental development scores. J of Ped 1983; 102: 519-22.

11. Lozoff B, Jiménez E, Wolf AW. Long term developmental outcome of infants with iron deficiency. N Eng J Med 1991; 325: 687-95.

12. Pollitt E. Effects of a diet deficient in iron on the growth and development of preschool and school-age children. Food Nut Bull 1991; 13: 110-8.

13. Walter T, et al. Effect of iron therapy on phagocytosis and bactericidal activity in neutrophils of iron deficient infants. Am J Clin Nutr 1986; 44: 877-82.

14. Chandra RK, Saraya AK: Impaired immunocompetence associated with iron deficiency. J Ped 1975; 86: 899902.

15. Beard JL, Borel M: Thermogenesis and iron deficiency anaemia. Nutr Tod 1988; 23: 41-5.

16. Dillman E, et al: Hypothermia in iron deficiency due to altered triiodothyronine metabolism. Am J Physiol 1980; 239: R377-81.

17. Dufour C, Brisigotti M, Fabretti G, Luxardo P, Mori $P G$, Barabino A: Helicobacter pylori gastric infection and sideropenic refractory anemia. J Pediatr Gastroenterol Nutr 1993; 17: 225-7.

18. Marignani $M$, Angeletti S, Bordi $C$, et al: Reversal of long-standing iron deficiency anaemia after eradication of Helicobacter pylori infection. Scand J Gastroenterol 1997; 32: 617-22.

19. Barabino A, Dufour C, Marino CE, Claudiani F, De Alessandri A: Unexplained refractory iron-deficiency anemia associated with Helicobacter pylori gastric infection in children: further clinical evidence. J Pediatr Gastroenterol Nutr 1999; 28: 116-9.

20. Annibale B, Marignani M, Monarca B, et al: Reversal of iron deficiency anemia after Helicobacter pylori eradication in patients with asymptomatic gastritis. Ann Intern Med 1999; 131: 668-72.

21. Milman N, Rosenstock S, Andersen L, Jorgensen T, Bonnevie $O$ : Serum ferritin, hemoglobin, and Helicobacter pylori infection: a seroepidemiologic survey comprising 2794 Danish adults. Gastroenterology 1998; 115: 26874.

22. Peach HG, Bath NE, Farish SJ: Helicobacter pylori infection: an added stressor on iron status of women in the community. Med J Aust 1998; 169: 188-90.

23. Collett JA, Burt MJ, Frampton CM, et al: Seroprevalence of Helicobacter pylori in the adult population of Christchurch: risk factors and relationship to dyspeptic 
symptoms and iron studies. N Z Med J 1999; 112: $292-$ 5 .

24. Choe YH, Lee JE, Kim SK: Effect of Helicobacter pylori eradication on sideropenic refractory anaemia in adolescent girls with Helicobacter pylori infection. Acta Pediatr 2000; 89: 154-7.

25. Barabino A: Helicobacter pylori-Related Iron Deficiency Anemia: A. Review. Helicobacter 2002; 7: 71-5.

26. Choe YH, Kim SK, Son BK, et al: Randomized placebocontrolled trial of Helicobacter pylori eradication for iron-deficiency anemia in preadolescent children and adolescents. Helicobacter 1999; 4: 135-9.

27. Sarker SA, Davidsson L, Mahmud H, et al: Helicobacter pylori infection, iron absorption, and gastric acid secretion in Bangladeshi children. Am J Clin Nutr 2004; 80: 149-53.

28. Mahalanabis, et al: Haematological response to iron supplementation is reduced in children with asymptomatic Helicobacter pylori infection. Br J Nutr 2005; 94: 969-75.

29. Gessner, et al: A controlled, household-randomized, open-label trial of the effect that treatment of Helicobacter pylori infection has on iron deficiency in children in rural Alaska. J I D 2006; 193: 537-46.

30. Seo, et al: Serum ferritin and Helicobacter pylori infection in children: a sero-epidemiologic study in Korea. J Gastroenterol Hepatol 2002; 17: 754-7.

31. Emin Kurekci A, Avni Atay A, Umit Sarici S, et al: Is there a relationship between childhood Helicobacter pylori infection and iron deficiency anemia? Trop Pediatr 2005; 51: 166-9.

32. Yang YJ, Sheu BS, Lee SC, Yang HB, Wu JJ: Children of Helicobacter pylori-infected dyspeptic mothers are predisposed to $H$. pylori acquisition with subsequent iron deficiency and growth retardation. Helicobacter 2005; 10: 249-55.

33. López T: Role of Helicobacter pylori infection in iron requirements or anaemia of hemodialysis patients. Nefrologia 2006; 26 (6): 673-8.

34. Baggett HC, Parkinson AJ, Muth PT, Gold BD, Gessner $B D$ : Endemic iron deficiency associated with Helicobacter pylori infection among school-aged children in Alaska. Pediatrics 2006; 117: e396-404.

35. Yuan W, Li Yumin, Yang Kehu, et al: Iron deficiency anemia in Helicobacter pylori infection: meta-analysis of randomized controlled trials. Scandinavian Journal of Gastroenterology 2010; 45: 665-76.

36. Zhang Z, Yang N, Zhao G, Zhu L, Zhi Y, Wang L: Effect of Helicobacter pylori eradication on iron deficiency. Chin Med J 2010; 123: 1924-30.
37. Xiaolu H, Xinhua Q, Weili $Y$, et al: Iron deficiency anaemia can be improved after eradication of Helicobacter pylori. Postgrad Med J 2010; 86: 272-27.

38. Malyszko J, Mysliwiec M: Hepcidin in Anemia and Inflammation in Chronic Kidney Disease. Kidney Blood Press Res 2007; 30: 15-30.

39. Fleming $R$, et al: Orchestration of iron homeostasis. N Eng1 J Med 2005; 352: 1741-4.

40. Lee SY, Song EY, Yun YM, et al: Serum prohepcidin levels in Helicobacter pylori infected patients with iron deficiency anemia. Korean J Intern Med 2010; 25: 195 200.

41. Cherian S, Forbes DA, Cook AG, et al: An insight into the relationships between hepcidin, anemia, infections and inflammatory cytokines in pediatric refugees: a cross-sectional study. PLoS One 2008; 3: e4030.

42. Schwarz P, Kübler JA, Strnad P, et al: Hepcidin is localised in gastric parietal cells, regulates acid secretion and is induced by Helicobacter pylori infection. Gut 2012; 61: 193-201.

43. Weiss $G$ : Iron metabolism in the anemia of chronic diseases. Biochem Biophys Acta 2009; 1790: 682-93.

44. Choe YH, Oh YJ, Lee NG, et al: Lactoferrin sequestration and its contribution to iron-deficiency anemia in Helicobacter pylori-infected gastric mucosa. J Gastroenterol Hepatol 2003; 18: 980-5.

45. Kassarjian Z, Russell RM: Hypochlorhydria: a factor in nutrition. Annu Rev Nutr 1989; 9: 271-85.

46. Charlton RW, Bothwell TH: Iron absorption. Annu Rev Med 1983; 34: 55-68.

47. Annibale B, Capurso G, Martino G, et al: Iron deficiency anaemia and Helicobacter pylori infection. Int J Antimicrobial Agents 2000; 16: 515-9.

48. Guiraldes E, Duarte I, Peña A, et al: Proinflammatory cytokine expression in gastric tissue from children with Helicobacter pylori associated gastritis. JPGN 2001; 33: 127-32.

49. Prinz C, Neumayer N, Mahr S, Classen M, Schepp W: Functional impairment of rat enterochromaffin-like cells by interleukin 1 beta. Gastroenterology 1997; 112: 36475.

50. Schepp W, Dehne K, Herrmuth H, Pfeffer K, Prinz $C$ : Identification and functional importance of IL-1 receptors on rat parietal cells. Am J Physiol 1998; 275: G1094-105.

51. Neu B, Randlkofer P, Neuhofer M, et al: Helicobacter pylori induces apoptosis of rat gastric parietal cells. Am. J. Physiol. Gastrointest. Liver Physiol 2002; 283: G30918.

52. El-Omar EM, Oien K, El-Nujumi A, et al: Helicobacter 
pylori infection and chronic gastric acid hyposecretion. Gastroenterology 1997; 113: 15-24.

53. Furuta T, El-Omar EM, Xiaoa F, Shirai N, Takashima $M$, Sugimurra $H$ : Interleukin 1 polymorphisms increase risk of hypochlorhydria and atrophic gastritis and reduce risk of duodenal ulcer recurrence in Japan. Gastroenterology 2002; 123: 92-105.

54. Atkinson S, Rockett K, Morgan G: Tumor necrosis factor SNP haplotypes are associated with iron deficiency anemia in West African children. Blood 2008; 112: 4276-83.

55. Weinber E: Iron availability and infection. Biochem. Biophys. Acta 2009; 1790: 600-5.

56. Belzer C, Stoof J, van Vliet A: Metal-responsive gene regulation and metal transport in Helicobacter species. Biometals 2007; 20: 417-29.

57. Senkovich O, Ceaser S, McGee DJ, Testerman TL: Unique host iron utilization mechanisms of Helicobacter pylori revealed with iron-deficient chemically defined media. Infect. Immun 2010; 78: 1841-9.

58. Huynh HQ, Campbell MA, Couper RT, Tran CD, Lawrence A, Butler RN: Lactoferrin and desferrioxamine are ineffective in the treatment of Helicobacter pylori infection and may enhance $H$. pylori growth and gastric inflammation in mice. Lett Appl Microbio 2009; 48: 517-22.

59. Park SA, Lee HW, Hong MH, et al: Comparative proteomic analysis of Helicobacter pylori strains associated with iron deficiency anemia. Proteomics 2006; 6: 131928.

60. Lee JH, Choe YH, Choi YO: The expression of ironrepressible outer membrane proteins in Helicobacter pylori and its association with iron deficiency anemia. Helicobacter 2009; 14: 36-9.

61. Walter T, Olivares $M$, Hertrampf E: Field trials of food fortification with iron: the experience in Chile. In: Lonnerdal B, ed. Iron Metabolism in Childhood. CRC Press, Boca Ratón, Florida, 1990, p. 127-55. 